\section{Endocarditis infecciosa: características clínicas, complicaciones y mortalidad en 506 pacientes y factores pronósticos de sobrevida a 10 años (1998-2008). Estudio cooperativo nacional en endocarditis infecciosa en Chile (ECNEI-2)}

\author{
MIGUEL OYONARTE ${ }^{1}$, RODRIGO MONTAGNA ${ }^{2}$, SANDRA BRAUN $^{3}$, \\ PAMELA ROJO ${ }^{4}$, JOSÉ L. JARA ${ }^{2}$, MAURICIO CERECEDA ${ }^{5}$, \\ MARCELO MORALES ${ }^{6}$, CAROLINA NAZAL $^{\mathrm{a}}$, FAUSTINO ALONSO ${ }^{7}$
}

\section{Clinical characteristics, complications and mortality in 506 patients with infective endocarditis and determinants of survival rate at 10 years}

Background: Rates of morbidity and mortality in Infective Endocarditis (IE) remain high and prognosis in this disease is still difficult and uncertain. Aim: To study IE in Chile in its active phase during inpatient hospital stay and long term survival rates. Material and Methods: Observational prospective national cohort study of 506 consecutive patients included between June 1, 1998 and July 31, 2008, from 37 Chilean hospitals (secondary and tertiary centers) nationwide. Results: The main findings were the presence of Rheumatic valve disease in $22.1 \%$ of patients, a history of intravenous drug abuse (IVDA) only in 0.7\%, the presence of Staphylococcus aureus in 29.2\% of blood cultures, negative blood cultures in 33.2\%, heart failure in $51.7 \%$ and native valve involvement in $86 \%$ of patients. Echocardiographic diagnosis was achieved in 94\% of patients. Hospital mortality was $26.1 \%$ and its prognostics factors were persisting infection (Odds ratio $(O R)$ 6.43, Confidence Interval (CI) 1.45-28.33\%), failure of medical treatment and no surgical intervention (OR 48.8; CI 6.67-349.9). Five and 10 years survival rates were 75.6 and $48.6 \%$, respectively. The significant prognostic factors for long term mortality, determined by multivariate analysis were the presence of diabetes, Staphylococcus aureus infection, sepsis, heart failure, renal failure and lack of surgical treatment during the IE episode. Conclusions: The microbiologic diagnosis of IE must be urgently improved in Chile. Mortality rates are still high (26.1\%) partly because of a high incidence of negative blood cultures and the need for more surgical valve interventions during in-hospital period. Long term prognostic factors for mortality should be identified early to improve outcome.

(Rev Med Chile 2012; 140: 1517-1528).

Key words: Bacteremia; Endocarditis, bacterial; Mortality; Staphylococcus aureus.

\author{
${ }^{1}$ Centro Cardiovascular, \\ Hospital Clínico San Borja \\ Arriarán, MINSAL. Facultad \\ de Medicina, Campus \\ Centro, Universidad de \\ Chile. \\ 2Departamento de \\ Cardiología Clínica \\ Alemana de Santiago. \\ Facultad de Medicina \\ Universidad del Desarrollo. \\ ${ }^{3}$ Departamento \\ de Enfermedades \\ Cardiovasculares. \\ Facultad de Medicina y \\ Hospital Clínico Pontificia \\ Universidad Católica de \\ Chile. \\ ${ }^{4}$ Departamento de \\ Cardiología Clínica Dávila. \\ ${ }^{5}$ Departamento \\ Cardiovascular, Hospital \\ Clínico José Joaquín \\ Aguirre. Facultad de \\ Medicina, Campus Norte, \\ Universidad de Chile. \\ ${ }^{6}$ Departamento de \\ Cardiología Hospital San \\ Juan de Dios. MINSAL. \\ Facultad de Medicina, \\ Campus Occidente, \\ Universidad de Chile. \\ ${ }^{7}$ Escuela de Salud Pública. \\ Facultad de Medicina, \\ Universidad de Chile. \\ aEnfermera universitaria, \\ Magíster en Salud Pública. \\ Facultad de Medicina, \\ Universidad de Chile. \\ Sin fuente de \\ financiamiento. \\ Recibido el 7 de mayo \\ 2012, aceptado el 24 de \\ agosto de 2012 . \\ Correspondencia a: \\ Dr. Miguel Oyonarte \\ Hernando de Aguirre 194 \\ Of. 62. Providencia. \\ Santiago. \\ E-mail:miguel.oyonarte@ \\ mi.cl
}


L a endocarditis infecciosa (EI) tiene una alta mortalidad entre las enfermedades cardiovasculares; ella ha constituido un desafío permanente para la medicina moderna por más de una centuria ${ }^{1-4}$. Esta elevada mortalidad alcanza el $25 \%$ de los $\operatorname{casos}^{4-5}$, y al año de su alta hospitalaria en cifras vecinas al $40 \%{ }^{6}$. En EI por Pseudomona aeuriginosa, Enterobacteriaceas y hongos, esta mortalidad puede superar el 50\% de los casos durante el período intrahospitalario. En una reciente comunicación del grupo del Estudio Colaborativo Internacional de EI (ICE-PCS), la mortalidad durante el episodio agudo fue de $17,7 \%{ }^{5}$, a pesar de los avances crecientes en infectología, los altos estándares en aplicación para la técnica de los hemocultivos (automatización), los nuevos y poderosos antibióticos que se emplean en la actualidad en esta enfermedad ${ }^{6-10}$, de la incorporación de nuevas técnicas diagnósticas imagenológicas, entre las que se destaca, especialmente la ecocardiografía ${ }^{11-19}$; los nuevos y estrictos criterios diagnósticos que se han incorporado ${ }^{20-22}$ y el perfeccionamiento de las técnicas cardioquirúrgicas, incluidos rigurosos controles pre e intraoperatorios, así como de los dispositivos intracardiacos en uso ${ }^{23-27}$, todos ellos factores que se suman para reducir la morbimortalidad de esta patología en las últimas décadas ${ }^{28-30}$, esta mortalidad continúa elevada.

Con el objetivo de estudiar esta enfermedad en Chile, su expresión clínica, complicaciones, mortalidad y sobrevida a 10 años de su seguimiento, hemos diseñado un estudio prospectivo nacional en EI, desde 1988 al 2008: "Estudio Cooperativo Nacional de Endocarditis Infecciosa" (ECNEI), cuyos resultados preliminares fueron publicados el año 2003, referido a los primeros 321 pacientes consecutivos estudiados en esta cohorte (19982002) $)^{31}$.

La presente comunicación muestra las características clínicas en 506 casos (que incluye los 321 anteriores), su mortalidad intrahospitalaria (1998-2008) y el seguimiento a 10 años para determinar la sobrevida de ellos e identificar los factores pronósticos de mortalidad a largo plazo, en la fase final de este estudio.

\section{Pacientes y Método}

Nuestro grupo de estudio de EI, que forma parte del grupo de Estudios Multicéntricos de la Sociedad Chilena de Cardiología (SOCHICAR), condujo este estudio nacional cooperativo y prospectivo desde el 01 de junio de 1998 al 31 de julio de 2008. Pacientes de 37 hospitales chilenos (centros de salud secundarios y terciarios), fueron enrolados con la autorización previa del respectivo Jefe de Servicio de Medicina Interna de cada institución. Éstos incluyeron hospitales docentes asistenciales en Santiago y regiones, hospitales clínicos universitarios y de las Fuerzas Armadas, mayoritariamente hospitales del Ministerio de Salud (MINSAL), que atienden alrededor del $80 \%$ de la población chilena. Estos hospitales fueron agrupados a su vez de acuerdo con su localización geográfica en 3 áreas: Norte, Región Metropolitana (Santiago de Chile con aproximadamente el $40 \%$ de la población total del país) y $\mathrm{Sur}^{31,32}$. Comparar los resultados obtenidos en este estudio entre las 3 diferentes zonas definidas está fuera de los objetivos específicos de este trabajo y fue previamente comunicado en la primera etapa de ECNEI (1998-2002) ${ }^{31}$.

\section{Selección de pacientes}

Se enviaron a los 37 hospitales seleccionados de Arica a Magallanes y que aceptaron participar en este estudio, utilizando los criterios de Duke $^{20,21}$ y Duke modificado ${ }^{22}$ para la formulación del diagnóstico de EI, las guías de prevención y tratamiento para EI elaboradas y recomendadas por el grupo de estudio de $\mathrm{EI}^{33}$. Los pacientes fueron incorporados por los médicos a cargo de este estudio a nivel local, de acuerdo a protocolo ad-hoc a llenar en cada paciente. Los pacientes que cumplían con estos criterios diagnósticos para EI (definida en $91 \%$ de los casos y posible en $9 \%$ de ellos), fueron incluidos para estudio y seguimiento; aquellos pacientes con EI posible, fueron tratados de manera similar a los portadores de EI definida. Quinientos seis pacientes cumplieron estos criterios diagnósticos entre 1998 y 2008. Cada paciente tuvo sólo un episodio de EI. El período de seguimiento finalizó el 2008.

\section{Registro de datos}

Se envió un protocolo similar para el registro de datos médicos a todos los centros, para la consignación de los hallazgos demográficos y clínicos, basados en la historia clínica del paciente, de acuerdo con definiciones previamente estan-

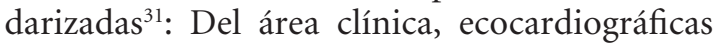
(ecocardiograma transtorácico-ETT y transeso- 
fágico-ETE), microbiológicos, complicaciones, tratamiento y la mortalidad. Estas definiciones fueron previamente publicadas en relación a nuestros primeros 321 pacientes estudiados ${ }^{31}$. Una de ellas, sepsis, se consideró como infección persistente (infección local no controlada y manifestaciones de infección extracardiacas); las formas aguda y subaguda, fueron consideradas en base al tiempo de evolución de esta entidad, de la severidad e intensidad de las manifestaciones clínicas y de su progresión desde el inicio de ella y previa a su tratamiento. Insuficiencia renal se definió en aquellos casos con creatinina plasmática $>1,5$ $\mathrm{mg} \%$. EI valvular prótesica (EIVP) precoz e EIVP tardía se analizan en su totalidad y no se estudian por separado.

La información recogida en cada hospital fue enviada al centro coordinador en el grupo de Estudio Multicéntrico de la SOCHICAR y analizados y tabulados por 2 de los investigadores principales. Luego del procesamiento de estos datos registrados, aquellos casos sin consistencia interna estadística fueron excluidos $(n=4 ; 0,8 \%)$.

\section{Seguimiento}

No fue posible obtener en 4 de los 506 pacientes información certera sobre su condición vital al finalizar el período intrahospitalario de la enfermedad. Por lo tanto, la mortalidad intrahospitalaria de este período, fue calculada sobre 502 casos. De los 371 pacientes que sobreviven al episodio de EI y que fueron dados de alta, posteriormente, en el tiempo de seguimiento, su condición o estado vital (vivo o muerto) se obtuvo de la búsqueda en el Registro Civil de Identificación, a través de su número nacional de identificación (RUN).

No fue posible obtener información on line sobre el tiempo de sobrevida en 43 pacientes, debido a que la información sobre su identificación (RUN) fue incompleta o equívoca.

El grupo de 43 pacientes perdidos en el seguimiento tienen características demográficas semejantes al grupo total analizado. Fueron excluidos en el análisis del seguimiento.

Así, en el análisis del seguimiento se consideran 328 pacientes ( $88,4 \%$ del total de pacientes) (Figura 1).

\section{Análisis estadístico}

El análisis descriptivo fue realizado utilizando promedio \pm DE para variables cuantitativas $y$ porcentajes para variables cualitativas; $\chi^{2}$ y $t$ de Student se utilizaron para comparar las variables de acuerdo a su naturaleza; $\chi^{2}$ se utilizó para comparar tasas de mortalidad entre las diferentes válvulas según tratamiento médico y quirúrgico. La sobrevida a largo plazo fue estudiada empleando el método de Kaplan-Meier. El análisis multivariado fue a base de regresión logística y el test de riesgos proporcionales de Cox para identificar factores de mortalidad en el seguimiento. Los modelos multivariados se aplicaron utilizando las variables seleccionadas que estadísticamente estaban asociadas en el análisis univariado. La curva ROC ("reciever operating characteristic") se empleó para determinar el valor predictivo del modelo obteni-

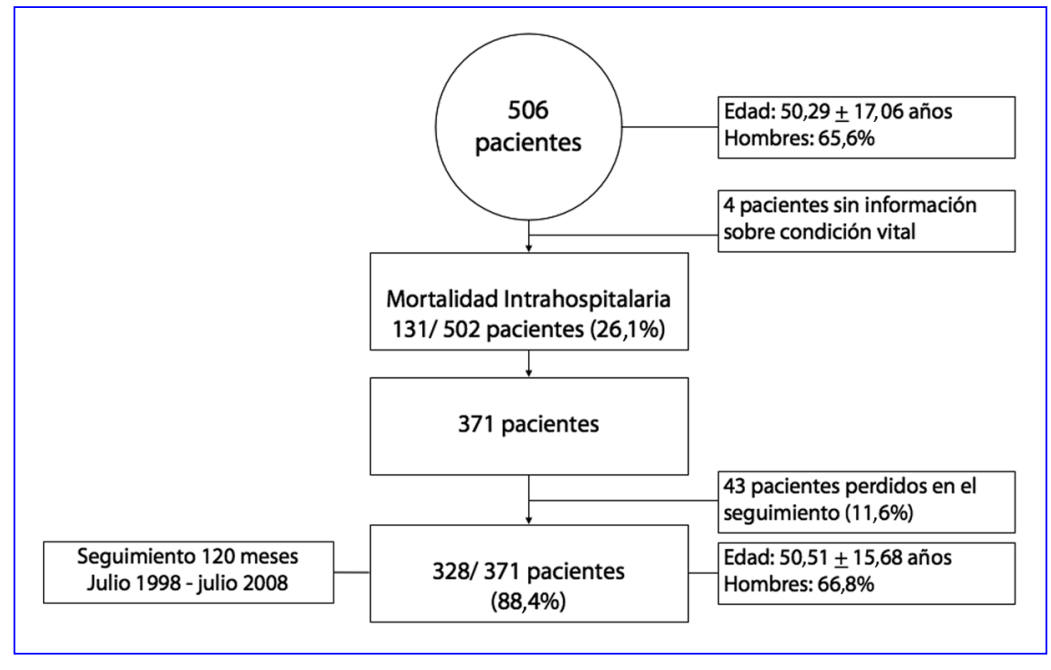

Figura 1. Representación de la población en estudio, incluyendo la fase intrahospitalaria y el período de seguimiento. 
do en el estudio multivariado de regresión logística. La significación estadística fue definida como $\mathrm{p} \leq 0,05$. Todo el análisis estadístico se hizo a través del Programa STATA 10 (Stata Corp. 2007. Stata Statistical Software: Release 10. College Station, TX: Stata Corp LP).

\section{Resultados}

Desde 1998 a 2008, un total de 506 pacientes fueron enrolados en el Estudio Prospectivo Nacional de Endocarditis Infecciosa, cumpliendo con los criterios de Duke para EI definida en $91,1 \%$ de los casos e EI posible en $8,9 \%$ de ellos.

En la Tabla 1 se presentan las variables demográficas, cardiopatías subyacentes, comorbilidades (o factores predisponentes de la enfermedad) y gérmenes aislados. La edad promedio fue 50,3 años (rango: 12-88 años) y $65,6 \%$ de la serie fueron varones. El tiempo promedio previo al diagnóstico de la enfermedad, desde el comienzo de los síntomas, fue de 36 días para la forma subaguda, identificándose ésta en el 63,1\% de los casos.

En cardiopatías subyacentes, la etiología reumática se encontró en $22,1 \%$ de los sujetos; válvulas protésicas en $14 \%$; cardiopatías congénitas 13,8\%; y ausencia de lesiones cardiacas en $26 \%$ de los casos. Se consignan la hemodiálisis $12,6 \%$ y diabetes mellitus $12 \%$, entre las comorbilidades más frecuentes. La drogadicción intravenosa $0,7 \%$, una vía excepcional en la drogadicción en Chile ${ }^{31}$.

Staphylococcus aureus, se hemocultivo en $29 \%$ de los casos estudiados (95/506); Streptococcus viridans en 22,9\% (75/506). Hemocultivos negativos en $33,2 \%(165 / 506)$ de los casos y sin información en esta serie en 2,17\% (11/506).

\section{Características clínicas}

Complicaciones, tratamiento médico, indicaciones quirúrgicas y desenlace (mortalidad y sobrevida), se encuentran en la Tabla 2. La insuficiencia cardiaca (IC) $(51,7 \%)$, el embolismo pulmonar y/o sistémico (30,6\%), la insuficiencia renal (IR)
(26,2\%), fueron encontrados entre las complicaciones más frecuentes. El tratamiento quirúrgico se efectuó en 35,3\% de la población estudiada. Las indicaciones más frecuentes (aisladas o en combinación), fueron las complicaciones intracardiacas: rotura valvular, perforación de velos, abscesos en $73,1 \%$ de los casos. IC en $63,6 \%$; infección persistente en $30,7 \%$. La mortalidad global intrahospitalaria fue $26,1 \%(131 / 502)$.

Tabla 1. Variables demográficas, condiciones cardiovasculares predisponentes, comorbilidades y microorganismos aislados en $\mathbf{5 0 6}$ pacientes con endocarditis infecciosa

\begin{tabular}{|c|c|c|}
\hline \multirow[t]{2}{*}{ Característica } & \multicolumn{2}{|c|}{ Hallazgo } \\
\hline & $\mathbf{n}$ & $(\%)$ \\
\hline \multicolumn{3}{|l|}{ Variables demográficas } \\
\hline Edad, promedio \pm DE años & \multicolumn{2}{|c|}{$50,2$ (rango: $12-88) \pm 17,0$} \\
\hline Hombres & 332 & 65,6 \\
\hline El Definida & 461 & 91,1 \\
\hline Período de latencia (días) & \multicolumn{2}{|c|}{$35,9$ (rango: $1-365) \pm 43,9$} \\
\hline Forma Subaguda & 286 & 63,1 \\
\hline \multicolumn{3}{|c|}{ Condiciones cardiovasculares predisponentes } \\
\hline Enfermedad reumática & 112 & 22,1 \\
\hline Prótesis valvular & 71 & 14,0 \\
\hline Cardiopatía congénita & 70 & 13,8 \\
\hline Degenerativa (esclerocálcica) & 41 & 8,1 \\
\hline Catéter intravenoso & 32 & 6,3 \\
\hline Mixomatosis & 22 & 4,3 \\
\hline Marcapasos & 16 & 3,1 \\
\hline Cirugía cardiaca previa & 10 & 1,9 \\
\hline Sin evidencia de cardiopatía & 132 & 26,0 \\
\hline \multicolumn{3}{|l|}{ Comorbilidades } \\
\hline Hemodiálisis & 64 & 12,6 \\
\hline Drogadicción intravenosa & 4 & 0,7 \\
\hline HIV positivo & 2 & 0,3 \\
\hline Diabetes mellitus & 61 & 12,0 \\
\hline Inmunosupresores & 8 & 1,5 \\
\hline Endocarditis infecciosa previa & 11 & 2,1 \\
\hline \multicolumn{3}{|l|}{ Microorganismos aislados } \\
\hline Staphylococcus aureus & 95 & 29,0 \\
\hline Streptococcus grupo & 75 & 22,9 \\
\hline Otros Streptococcus & 69 & 21,1 \\
\hline Estáfilococo coagulasa negativo & 36 & 11,0 \\
\hline Enterococo & 24 & 7,3 \\
\hline Bacilos Gram negativos & 17 & 5,2 \\
\hline Hongos & 6 & 1,8 \\
\hline HACEK & 5 & 1,5 \\
\hline Hemocultivos negativos & 168 & 33,2 \\
\hline Sin información & 11 & 2,1 \\
\hline
\end{tabular}


Tabla 2. Complicaciones, tipo de tratamiento, indicaciones de cirugía y pronóstico en $\mathbf{5 0 6}$ pacientes con Endocarditis Infecciosa (período intrahospitalario)

\begin{tabular}{|lcc|}
\hline Característica & \multicolumn{2}{c|}{ Hallazgo } \\
& n & (\%) \\
Complicaciones & & \\
$\quad$ Insuficiencia cardiaca & 262 & 51,7 \\
Embolismo (sistémico, pulmonar) & 155 & 30,6 \\
Insuficiencia renal & 133 & 26,2 \\
Tipo de tratamiento & & \\
Médico (conservador) & 273 & 53,9 \\
Quirúrgico & 179 & 35,3 \\
Indicaciones de cirugía\# & & \\
Complicaciones intra cardiacas & 131 & 73,1 \\
(rotura, perforación, abscesos) & 114 & 63,6 \\
Insuficiencia cardiaca & 55 & 30,7 \\
Infección persistente & 53 & 29,6 \\
Vegetaciones > 10 mm & 18 & 10,0 \\
Disfunción de prótesis valvular & 17 & 9,4 \\
Embolismo múltiple & 4 & 2,2 \\
Endocarditis de marcapasos & & \\
Pronóstico * & 131 & 26,1 \\
Fallece & 371 & 73,9 \\
Sobrevida & 28 & 15,6 \\
Mortalidad quirúrgica & & \\
\hline
\end{tabular}

\#Una o más indicaciones en algunos pacientes. *Calculado sobre 502 pacientes (4 de los 506 pacientes sin datos completos en relación a su estado vital).
La presencia de vegetaciones, abscesos y rotura valvular diagnosticadas por ecocardiografías ( $97 \%$ con ETE), se aprecian en la Figura 2, con cifras de $85,5 \%$ en valvular nativas y $14,4 \%$ de compromiso en valvular protésicas $(12,8 \%$ prótesis valvulares mecánicas y $1,6 \%$ en válvulas protésicas biológicas). El compromiso aórtico y mitroaórtico fue más frecuente en válvulas nativas en el ecocardiograma $(90,3 \%)$. La EI en válvulas protésicas mecánicas en posición mitral fue la más frecuente localización entre las endocarditis protésicas $(20 \%)$.

La mortalidad intrahospitalaria, de acuerdo a la válvula afectada, se aprecia en la Figura 3, con o sin tratamiento quirúrgico en 502 pacientes (análisis univariado); con el tratamiento quirúrgico la mortalidad para las válvulas afectadas en el corazón izquierdo disminuyó significativamente $(\mathrm{p}=0,003)$. La cirugía combinada mitral y aórtica tuvo una significativa mayor mortalidad que la aislada univalvular. La mortalidad global quirúrgica fue de $15,6 \%$ (27/177), en toda la serie.

La infección persistente y el fracaso del tratamiento médico antibiótico, sin terapia quirúrgica asociada, fueron ambos, los únicos factores pronósticos de mortalidad

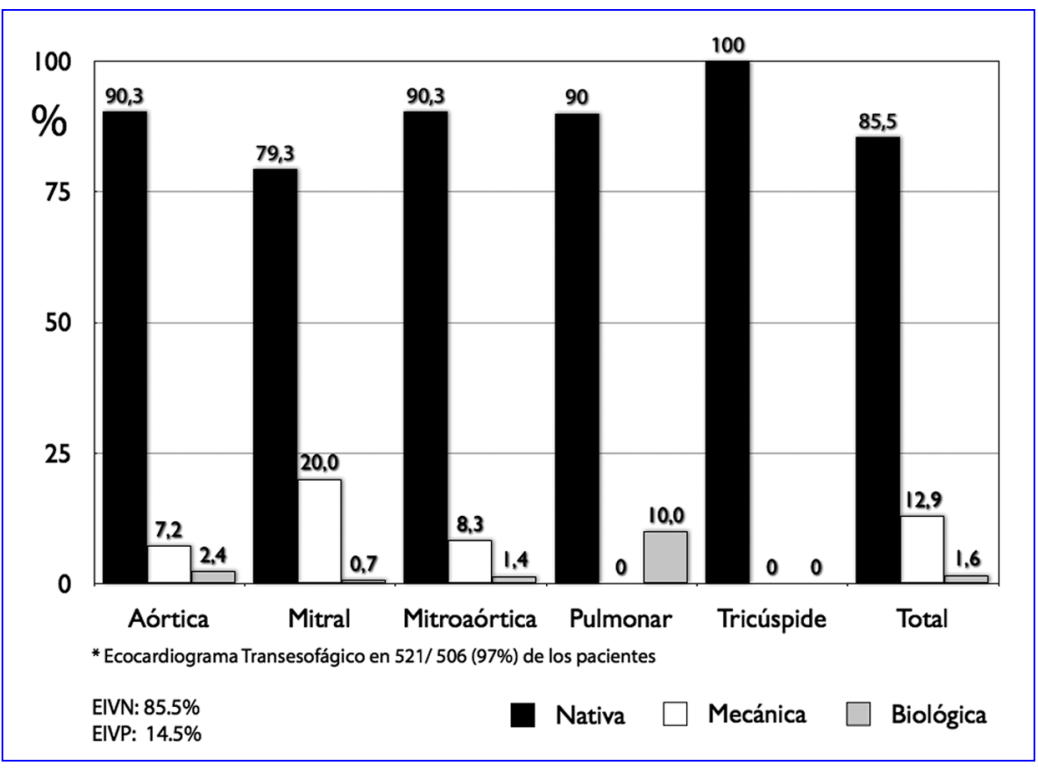

Figura 2. Tipo de válvula afectada por El de acuerdo a localización anatómica según diagnóstico ecocardiográfico (vegetación, absceso, disrupción valvular) en 506 pacientes del estudio ECNEI. EIVN: Endocarditis Infecciosa de válvula nativa. EIVP: Endocarditis infecciosa de válvula protésica. 


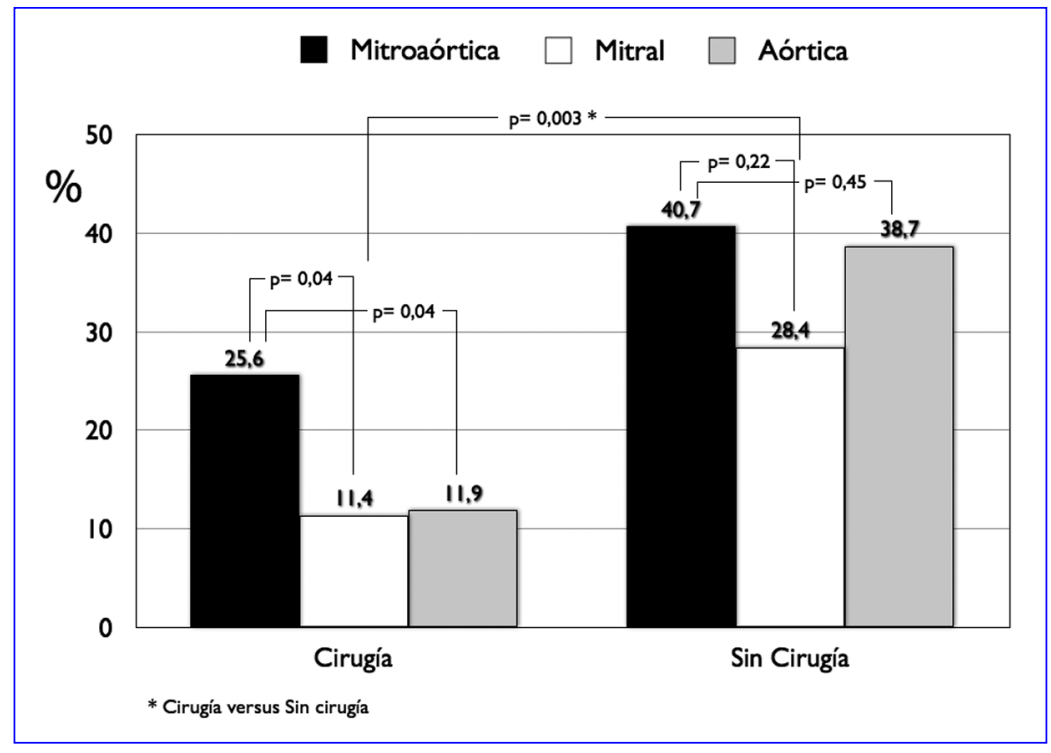

Figura 3. Mortalidad intrahospitalaria con y sin tratamiento quirúrgico de acuerdo a la válvula afectada en 502 pacientes del estudio ECNEI.

Tabla 3. Análisis multivariado de los factores de riesgo de la mortalidad intra hospitalaria en 502 pacientes con El estudiados durante el período 1998-2008

\begin{tabular}{|lccc|}
\hline Variable & OR & OR IC 95\% & p value \\
\hline Edad & 1,01 & $0,97-1,06$ & 0,384 \\
Género femenino & 1,55 & $0,37-6,49$ & 0,546 \\
Insuficiencia renal & 6,06 & $0,81-45,4$ & 0,079 \\
\hline Insuficiencia cardiaca & 0,26 & $0,05-1,23$ & 0,09 \\
Infección persistente & 6,43 & $1,45-28,33$ & 0,014 \\
Diabetes mellitus & 0,36 & $0,05-2,53$ & 0,051 \\
Staphylococcus aureus & 0,85 & $0,18-3,91$ & 0,844 \\
Compromiso mitroaórtico & 2,04 & $0,44-9,32$ & 0,355 \\
\hline Fracaso tratamiento médico y sin tratamiento quirúrgico & 48,82 & $6,67-349,97$ & $<0,001$ \\
\hline Embolismo & 0,46 & $0,10-1,94$ & 0,292 \\
\hline
\end{tabular}

Tabla 4. Modelo de riesgos proporcionales de Cox para factores pronósticos de mortalidad a largo plazo en 328 pacientes (en seguimiento) con El estudiados durante el período 1998- 2008

\begin{tabular}{|lccc|}
\hline Variable & $\begin{array}{c}\text { Hazard } \\
\text { Ratio }\end{array}$ & [95\% IC] & p value \\
\hline Diabetes mellitus & 1,68 & $1,05-2,69$ & 0,030 \\
Infección persistente & 1,88 & $1,28-2,77$ & 0,001 \\
\hline Staphylococcus aureus & 1,50 & $1,05-2,17$ & 0,026 \\
\hline Insuficencia cardiaca & 2,01 & $1,36-2,98$ & 0,001 \\
\hline Insuficiencia renal & 1,53 & $1,03-2,29$ & 0,037 \\
\hline Cirugía episodio agudo & 0,45 & $0,29-0,71$ & 0,001 \\
\hline
\end{tabular}

intrahospitalaria en esta serie (análisis multivariado), como se observa en la Tabla 3 y se valida con curva ROC, Figura 4.

\section{Seguimiento}

La curva de sobrevida para los 328 pacientes que sobrevivieron la fase hospitalaria se aprecia en la Figura 5. El porcentaje de sobrevida con una media de $61,1 \pm 35,5$ meses de seguimiento (rango $1-122,6$ meses) fue a 5 y 10 años, de $75,6 \%$ y $48,6 \%$ respectivamente.

Los factores pronósticos de mortalidad para este período de seguimiento: presencia de diabetes ( $\mathrm{p}<0,001)$, infección por Staphylococcus aureus 


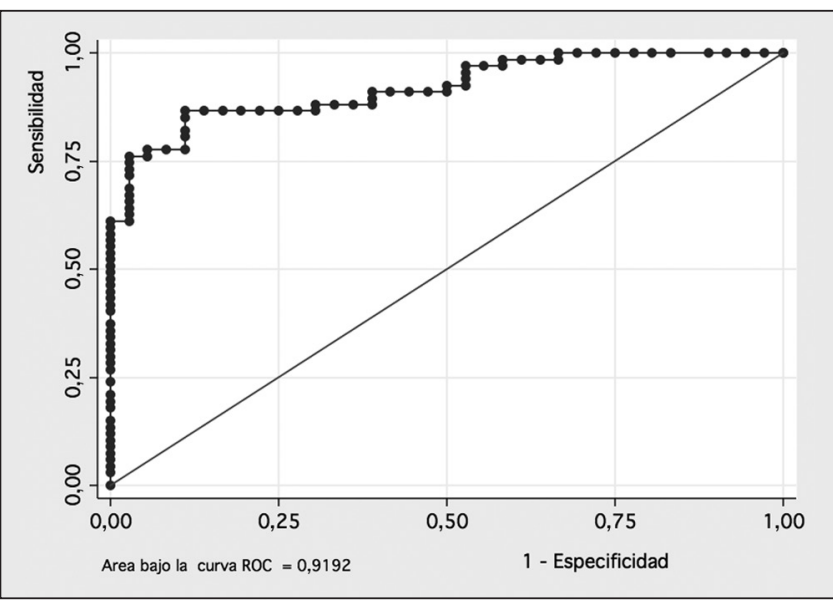

Figura 4. Curva ROC asociada al análisis multivariado de factores predictores de mortalidad en $\mathrm{El}$, en 502 pacientes estudiados durante el período 1998-2008 (mortalidad intrahospitalaria).

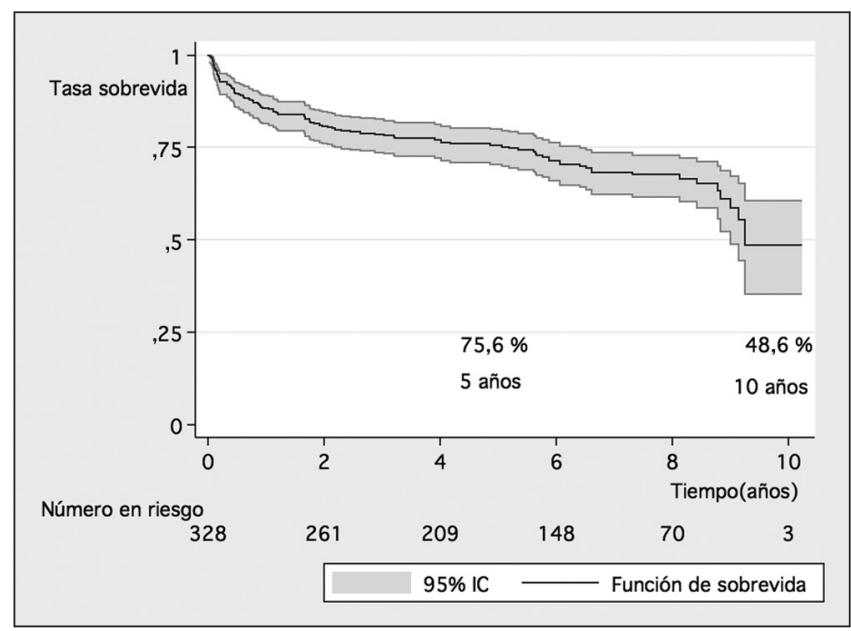

Figura 5. Curva de sobrevida de 328 pacientes con Endocarditis Infecciosa durante el período de estudio 1998-2008, estimada por el método de Kaplan-Meier. IC: intervalo de confianza.

( $\mathrm{p}=0,03)$, la infección persistente $(\mathrm{p}=0,001)$, la insuficiencia cardiaca $(\mathrm{p}=0,004)$, la insuficiencia renal $(\mathrm{p}=0,001)$, y la ausencia de tratamiento quirúrgico durante el período intrahospitalario $(\mathrm{p}<0,001)$, como se aprecia en la Tabla 4.

\section{Discusión}

\section{Demografía, factores predisponentes $y$} microbiología (período intrahospitalario)

La EI tiene una incidencia de aproximadamente 1,76,2 por 100.000 pacientes por año, con un incremento actual de la edad promedio y con una relación hombre/mujer de 2:1,34,35. Su incidencia en Chile está en alrededor de 2 casos por 100.000 pacientes por año en el área central de Santiago ${ }^{31}$; la evidencia de EI por narcoadicción intravenosa es casi anecdótica en este estudio, con una incidencia de $0,7 \%$, ya que esta forma de adicción es infrecuente en nuestro país ${ }^{31}$.

La edad de presentación va en aumento, con un promedio de 50,2 años (Tabla $1)$; en anteriores trabajos prospectivos nacionales, en el período 1980-1996, se observaba una media de 39 años $^{36}$. Dos tercios de nuestros pacientes en esta serie actual son varones, un número cercano al 68\% que se registró en el Estudio ICE ${ }^{5}$. La forma subaguda aún predomina en el $63,3 \%$ de los sujetos estudiados, lo habitual en este país. La cardiopatía reumática ha disminuido de $25,3 \%{ }^{31}$ al $22,1 \%$ en la fase final de este estudio cooperativo nacional, marcando la misma tendencia que se observa en países de alto desarrollo en que esta enfermedad ha disminuido significativamente.

Las válvulas protésicas, cardiopatías congénitas, cardiopatías degenerativas esclerocálcicas y marcapasos cardiacos, son las otras patologías cardiacas de base, que van en paralelo a los cambios epidemiológicos ocurridos en los últimos tres a cuatro decenios ${ }^{4-8}$. La ausencia de daño cardiaco estructural en enfermos con EI, se observó en $26,1 \%$ de ellos en el examen ecocardiográfico. En otros estudios prospectivos la ausencia de daño cardiaco previo, se observa en $21 \%$, $35,1 \%$ y $38,6 \%$ respectivamente durante períodos similares de estudio ${ }^{37-39}$.

La EIVP se encuentra en 7 a 25\% de los casos en países desarrollados ${ }^{14}$; en este estudio se encuentra en $14,5 \%$ de la serie, cifra menor a la publicada en el Estudio ICE que registró esta patología en diferentes países, con distintos grados de desarrollo socioeconómico en $21 \%{ }^{5}$.

En los pacientes con insuficiencia renal y en hemodiálisis $(12,6 \%)$ y con diabetes (12\%), aumenta la incidencia de EI a través de la disminución de los 
mecanismos de inmunidad, siendo estos pacientes muy susceptibles a contraer infecciones estafilocócicas, ya que están más expuestos a procedimientos invasivos, tanto en centros hospitalarios como de la comunidad ${ }^{5,6,42}$.

Hemos observado un cambio en las características de los gérmenes causales de EI en nuestros hospitales, con $29 \%$ de infecciones por Staphylococcus aureus aislados en hemocultivos y $23 \%$ por el grupo Viridans. Estos resultados confirman publicaciones internacionales previas ${ }^{4-6,37,40,41}$, incluyendo infecciones (hemocultivos) no nosocomiales, en centros de salud externos en EI valvular nativa ${ }^{42}$ y muestra un viraje bacteriológico local, si se considera que el Streptococcus viridans se aisló en $31 \%$ de los casos y el Staphylococcus aureus en 18\%, en un estudio nacional con 261 pacientes evaluados entre 1980-1999, en un hospital universitario de Santiago ${ }^{43}$. En Argentina, en el Estudio EIRA-2 (EI en la República Argentina), estudio nacional cooperativo y prospectivo, se describe en el área de la microbiología una incidencia de 29,8\% para Staphylococcus aureus y de $27 \%$ para Streptococcus viridans ${ }^{38}$. Para todo el grupo de Streptococcus, la incidencia global fue $38,3 \%$ y para el grupo Estafilocócico de 36,7\%, comparado con nuestra serie en que para todo el grupo Estreptocócico, la incidencia fue $44,1 \%$ y para el Estafilococico 40,06\% (Tabla 1). De este modo, en estos dos países vecinos en Sudamérica, el Staphylococcus aureus, es el agente causal aislado más frecuentemente en esta enfermedad. Algo similar se ha comunicado en Brasil en un centro terciario de salud ${ }^{44}$.

Los hemocultivos negativos $(33,2 \%)$ se explican probablemente por el empleo previo de antibióticos $^{4,45}$ y en Chile, esta elevada cifra de negatividad, puede explicarse también porque la técnica automatizada para procesar los hemocultivos no está lo suficientemente extendida en los laboratorios del país siendo, en muchas ocasiones, analizados mediante la técnica manual, lo que condiciona una menor sensibiliadad ${ }^{46}$. Los estudios serológicos y las técnicas moleculares de estudio para ácidos nucleicos del germen causal, a través de la reacción de polimerasa en cadena $(\mathrm{PCR})^{47,48}$, están comenzando a incluirse y difundirse en la práctica clínica, con lo que se espera contribuya a disminuir el número de EI sin reconocimiento de su agente causal. La baja positividad de los hemocultivos a nivel nacional es una cifra mayor a lo registrado para otros países de Sudamérica según el Estudio ICE ${ }^{5}$, lo que nos debe motivar a mejorar estas técnicas de estudio en forma masiva con políticas nacionales de salud, para corregir esta preocupante realidad sanitaria.

\section{Características clínicas, tratamiento y desenlace (Etapa intrahospitalaria)}

En esta cohorte de pacientes, la insuficiencia cardiaca $(51,7 \%)$, el embolismo $(30,6 \%)$ y la insuficiencia renal $(26,2 \%)$ son las tres complicaciones más frecuentes, algo similar a lo reportado en el ICE ${ }^{5}$. La cirugía se efectuó en 35,3\% de los casos (Tabla 2). Las complicaciones intracardiacas y la IC secundaria fueron la primera causa de indicación quirúrgica y representan a más de la mitad de las indicaciones para este tipo de tratamiento ${ }^{49-54}$. En el Estudio ICE la cifra de intervenciones quirúrgicas llegó al 48,2\% y la mortalidad global hospitalaria fue de $17,7 \%$, lo que contrasta con la de nuestra serie de 26,1\%. Como Kemose et al. ${ }^{54}$, hemos observado una significativa mayor mortalidad quirúrgica cuando se opera simultáneamente las válvulas afectadas mitral y aórtica, comparado con el reemplazo valvular individual (Figura 3 ).

A corto plazo en nuestro país nos parece que las indicaciones de cirugía precoz en esta entidad debieran aumentar y con ello posiblemente disminuiríamos esta mortalidad intrahospitalaria ${ }^{5}$, lo que sumado a un mayor porcentaje de hemocultivos positivos debieran optimizar el manejo antibiótico y médico-quirúrgico en la $\mathrm{EI}^{52,53}$, mejorando el pronóstico de estos pacientes. De ahí que no debe sorprender que como factores pronósticos de mortalidad encontramos fiebre persistente y fracaso del tratamiento antibiótico sin cirugía asociada en estudio multivariado para este período (Tabla 3 ), lo que se valida por curva ROC (Figura 4).

\section{Seguimiento}

La curva de Kaplan-Meier, a 5 años del alta, muestran una sobrevida de $75,6 \%$ y a 10 años, sólo de 48,6\% (Figura 5). Recaídas y recurrencias o la necesidad de una intervención quirúrgica luego del período intrahospitalario quedan fuera de los objetivos de este estudio, por lo que no se han consignado. Tornos et al. ${ }^{55}$, en su serie publicada en 1992, estudiando EI en pacientes sin narcoadicción, muestra una sobrevida a 5 y 10 años de $88 \%$ y $81 \%$ respectivamente. Su estudio incluye 
el registro de sobrevida a 15 años y que llegó al $61 \%$ de sobrevida. Estas cifras son muy distintas y mejores a 10 años de seguimiento, al compararlas con nuestros resultados. Sin embargo, el estudio de Netzer et al comunica resultados similares a nuestra serie ${ }^{56}$. Castillo et al. ${ }^{37}$ comunican $96 \%$ de sobrevida a 5 años para EI valvular nativa y de $82 \%$ para EIVP. La EI por drogadicción intravenosa no se incluye en estas dos series ${ }^{37,55}$. En nuestra casuística sólo tuvimos 4 casos de EI por narcoadicción intravenosa $(0,7 \%)$ por lo que estas dos series citadas pueden ser comparables con nuestra población. Intentando entonces explicar estos bajos porcentajes de sobrevida en nuestra cohorte de pacientes en el largo plazo, identificamos 6 factores predictivos de mortalidad mediante análisis multivariado: Diabetes $(\mathrm{p}<0,001)$, S. aureus ( $\mathrm{p}$ $=0,03)$; infección persistente $(\mathrm{p}=0,01)$; insuficiencia cardiaca $(p=0,04)$, insuficiencia renal ( $p$ $=0,001)$ y ausencia de tratamiento quirúrgico durante el período de estado de la enfermedad (intrahospitalario) $(\mathrm{p}<0,001)$ en Tabla 4. La identificación de estos factores pronósticos de mortalidad al momento del alta, podría determinar una conducta médica más activa y de alerta sobre posibles complicaciones en los grupos de referencia o de control y manejo de estos pacientes luego de su egreso hospitalario.

Hasbún et al. ${ }^{57}$ afirman que los pacientes adultos con EI de válvulas nativas en corazón izquierdo pueden ser estratificados acertadamente previo al alta para los factores asociados a mortalidad en los 6 meses de seguimiento. Los hallazgos clínicos a que alude son: estado mental anormal (confusión), IC moderada a severa, etiología bacteriana diferente a Streptococcus viridans y tratamiento médico sin cirugía, lo que se acerca a nuestros resultados.

Para estratificación de riesgo es ideal tener identificado el microorganismo causal y las complicaciones clínicas, para así mejor decidir el momento quirúrgico. En estudios retrospectivos recientes publicados en el medio nacional, referente a pacientes sometidos a cirugía en EI, que abarcan décadas del ochenta, noventa del siglo XX y comienzos del siglo XXI ${ }^{59-61}$, se evidencia en algunos de ellos la alta negatividad en hemocultivos: $47 \%$ para el grupo de Temuco ${ }^{60}$, con predominio de estafilococos; $51 \%$ de negatividad para el grupo de Concepción ${ }^{61}$, siendo el estafilococo el germen aislado más frecuente pero predominando como grupo os estreptococos. En el trabajo de Zalaquett et al., del grupo de Santiago ${ }^{59}$, por razones metodológicas, todos los paciente que incluye el trabajo tienen hemocultivos positivos, predominando el estreptococo. La causa más frecuente de complicación y de indicación quirúrgica fue la ICC: $50 \%{ }^{59}$, $65,6 \%{ }^{60}$ y $54,7 \%{ }^{61}$

El tratamiento quirúrgico en las tres series tiene buen resultado a corto y largo plazo ${ }^{59,60}$. Resultados todos comparables con nuestra serie aunque ECNEI es un estudio prospectivo y multicéntrico.

\section{Limitaciones del estudio}

Este trabajo corresponde al informe final de esta cohorte de pacientes con EI, estudiado prospectivamente a lo largo del país, por ello, tiene las limitaciones inherentes a los estudios observacionales. Los centros de referencia nacionales son heterogéneos en su estructura y nivel de complejidad: algunos secundarios, otros terciarios con diferentes grados de especialización y experiencia en el diagnóstico y manejo médico y quirúrgico aplicables a esta patología; especialmente notorio en el caso de la toma y procesamiento de los hemocultivos (técnica manual versus automatizada) y en la presencia y composición de los grupos cardioquirúrgicos, actores fundamentales para la toma de decisiones y elección del momento óptimo de estas intervenciones.

Sin embargo, muestra la real condición de esta enfermedad en algunos aspectos centrales de ella en Chile, su impacto en la salud de nuestra población y nos entrega evidencias que pueden aportar para mejorar las políticas públicas en este campo.

\section{Conclusiones}

En nuestro país el diagnóstico bacteriológico debe ser urgentemente mejorado a través de la implementación generalizada de las técnicas automatizadas para el estudio de los hemocultivos.

La mortalidad de EI es aún elevada $(26,1 \%)$, en parte explicada por la alta incidencia de hemocultivos negativos y un tratamiento antibiótico empírico en un $1 / 3$ de los casos y posiblemente por la ausencia de un mayor número de intervenciones quirúrgicas oportunas en el período intrahospitalario.

Los factores pronósticos de mortalidad a largo plazo, en el seguimiento, deben ser identificados 
para mejorar el desenlace y evolución clínica de ellos, facilitando una mejor labor entre cardiólogos, infectólogos y cardiocirujanos en el proceso de toma de decisiones clínicas ${ }^{48-54,58}$.

Agradecimientos: A la Sra. Ximena Bascur, secretaria de la SOCHICAR, por su extraordinario trabajo de coordinación administrativa a nivel nacional.

\section{Addendum}

Listado de Hospitales incorporados al estudio en Chile y de investigadores responsables en estudio ECNEI

Hospitales de regiones: Hospital Regional de Arica (Dr. Hernán Figueroa), Hospital Regional de Iquique (Dr. Ignacio Auger, Dra. Virginia Martínez), Hospital Regional de Antofagasta (Dr. Manuel Saavedra), Hospital Regional de La Serena/Coquimbo (Dr. Carlos Echeverría, Dr. Claudio Bugueño), Hospital Ovalle (Dr. Fernando Arab), Hospital San Camilo de San Felipe (Dra. Lía Bunster), Hospital Quilpué (Dr. Jaime Quilodrán), Hospital Gustavo Fricke Viña del Mar (Dr. Javier Alburquerque, Dr. Luis Becerra), Hospital Naval Viña del Mar (Dra. Marcela Ghiringhelli), Hospital Van Buren Valparaíso (Dr. Carlos Astudillo), Hospital Regional de Rancagua (Dr. Leopoldo Manríquez), Hospital San Fernando (Dr. Carlos Sáez), Hospital Regional de Talca (Dr. Enrique Mercadal, Dr. Patricio Marín), Hospital Herminda Martín de Chillán (Dr. Héctor Garay), Hospital Regional de Concepción (Dr. Hernán Carrillo, Dr. Carlos Otero), Hospital Víctor Ríos Ruíz Los Angeles (Dra. Mónica Fiedler), Hospital Regional de Temuco (Dr. Benjamín Stockins), Hospital Clínico de Valdivia (Dr. Juan C Bertoglio), Hospital Base de Osorno (Dr. Gabriel Jano Morales), Clínica Alemana Puerto Varas (Dr. Ricardo Vargas), Hospital de Castro (Dr. Claudio Santibáñez), Hospital Puerto Montt (Dr. Felipe del Campo), Hospital Coyhaique (Dr. Gustavo González), Hospital Regional de Punta Arenas (Dr. Guillermo Araneda).

Hospitales Área Metropolitana: Asistencia Pública (Dr. Livio Casorzo), Hospital Barros Luco Trudeau (Dra. Ligia Gallardo, Dr. Pablo Pedrero), Hospital Clínico Pontificia Universidad Católica de Chile (Dra. Sandra Braun), Hospital Clínico Universidad de Chile (Dr. Miguel Oyonarte G, Dr. Rodrigo Montagna), Hospital Dipreca (Dra.
Pamela Rojo), Hospital Fach (Dr. Rodrigo Hernández), Hospital Militar (Dr. Ricardo Morales), Hospital del Salvador (Dr. Claudio Parra), Hospital San Borja Arriarán (Dr. Alberto Estévez, Dr. Marcelo Lindh), Hospital San José (Dr. Armando Andrade, Dr. Luis Gamboa), Hospital San Juan de Dios (Dr. Leonel López), Hospital Sótero del Río (Dr. Isabel Hoffman), Hospital del Tórax (Dr. Polentzi Uriarte, Dr. Sergio Bernal).

\section{Referencias}

1. Major RM. Note on the History of Endocarditis. Bull Hist Med 1945; 17: 351-9.

2. Osler W. The Gulstonian Lectures, on malignat Endocarditis. Br Med J 1885; 1: 467-579.

3. Ledoux-Lebard. Patogenia i Anatomía Patológica de la endocarditis ulcerosa. Rev Med Chile 1886; 14: 231-5.

4. Mylonakis E, Calderwood SB. Infective endocarditis in adults N Engl J Med 2001; 345: 1318-30.

5. Murdoch DR, Corey GR, Hoen B, Miró JM, Fowler VG Jr, Bayer AS, et al. Clinical Presentation, Etiology and Outcome of Infective Endocarditis in the 21 st. Century. The International Collaboration on EndocarditisProspective Cohort Study. Arch Intern Med 2009; 169 (5): 463-73.

6. Cabell CH, Jollis JG, Peterson GE, Corey GR, Anderson DJ, Sexton DJ, et al. Changing Patient Characteristics and the Effect on Mortality in Endocarditis. Arch Intern Med 2002; 162 (1): 90-4.

7. Nissen H, Nielsen PF, Frederiksen M, Helleberg C, Nielsen JS. Native valve infective endocarditis in the general population: A 10 year survey of the clinical picture during 1980s. Eur heart J 1992; 13 (7): 872-7.

8. Benn M, Hagelskjaer LH, Tvede M. Infective endocarditis, 1984 through 1993; a clinical and microbiological survey. J Intern Med. 1997; 242 (1): 15-22.

9. Baddour LM, Wilson WR, Bayer AS, Fowler V Jr, Bolger A, Levinson M, et al. Infective endocarditis: Diagnosis, antimicrobial therapy, and complications. Circulation 2005; 111: 3167-84.

10. Bayer AS, Bolger AF, Taubert KA, Wilson W, Steckelberg J, Karchmer A, et al. Diagnosis and management of infective endocarditis and its complications. Circulation 1998; 2396-48.

11. Dillon JC, Feigenbaum H, Konecke LL, Davis RH, Chang S. Echocardiographic manifestation of valvular vegetations. Am Heart J 1973; 86: 698-704.

12. Mügge A, Werner ED, Frank G, Lichtien PR. Echocardiography of infective endocarditis. Reassessment of 
prognostic implication of vegetation size determined by the transthoracic and transesophageal approach. J Am Coll Cardiol 1989; 14: 631-8.

13. San Philippo AJ, Picard MH, Newell JB, Rosas E, Davidoff $\mathrm{R}$, Thomas J, Weyman A. Echocardiographic assessment of patients with infective endocarditis: Prediction of risk for complications. J Am Coll Cardiol 1991; 16: 1191-9.

14. Steckelberg JM, Murphy JG, Ballard D. Emboli in infective endocarditis. The prognostic value of echocardiography Ann Intern Med 1991; 114: 635-40.

15. Daniel WG, Mûgge A, Grote J, Hausmann D, Nikutta P, Laas J, Lichtlen PR, Martin R, et al. Comparison of transthoracic and transesophageal echocardiography for detection of abnormalities of prosthetic and bioprosthetic valves in mitral and aortic positions. Am J Cardiol 1993; 71: 210-5.

16. Vilacosta I, Sarria C, San Román JA, Jiménez J, Castillo JA, Iturralde E, et al. Usefulness of transesophageal echocardiography for diagnosis of infected transvenous permanent pacemakers. Circulation 1994; 89: 2684-7.

17. Jacob S, Tong T. Role of echocardiography in the diagnosis and management of infective endocarditis. Curr Op in Cardiol 2002; 17: 478-85.

18. Jain R, Kolias T. Three-Dimensional Transesophageal Echocardiography of Pacemaker Endocarditis. J Am Coll Cardiol 2009; 53: 1241.

19. Feuchtuer GM, Stolzmann P, Dichtl W, Schertler T, Bonatti J, Scheffel H, et al. Multi-slice computed tomography in infective endocarditis. J Am Coll Cardiol 2009; 53: 436-444.

20. Durack DT, Lukes AS, Bright DK. Duke Endocarditis Service. New criteria for diagnosis of infective endocarditis: Utilization of specific echocardiographic finding. Duke Endocarditis Service. Am J Med 1994; 96 (3): 2009.

21. Bayer AS, Ward TY, Winzton LE, Shapiro SM. Evaluation of new clinical criteria for the diagnosis of infective endocarditis. Am J Med 1994; 96: 211-2119.

22. Li JS, Sexton DJ, Mick N, Nettles R, Fowler VG Jr, Ryan $\mathrm{T}$, et al. Proposed modification to the Duke criteria for the diagnosis of infective endocarditis. Clin Infect Disease 2000; 30: 633-8.

23. Kay JH, Bernstein S, Feinstein D, Biddle M. Surgical cure of candida albicans endocarditis with open heart surgery. N Engl J Med 1961; 264: 907-10.

24. Mullany CJ, Mc Isaacs AI, Rowe MH, Hale GS. The surgical treatment of infective endocarditis. Word J Surg 1989; 13 (1): 132-6.

25. Drinkwater DC, Laks $\mathrm{H}$, Child J. Issues in surgical treatment of endocarditis including intra-operative and postoperative management. Cardiol Clinic 1996; 14: 451-64.

26. Acar J, Michel PL, Varenne O, Michaud D, Rafik T. Surgical treatment of infective endocarditis. Eur Heart J 1995; 16 (Suppl B) 94-8.

27. Klug D, Lacroix D, Savoye C, Goullard L, Grandmougin D, Hennequin JL, et al. Systemic infection related to Endocarditis of Pacemaker Leads. Clinical Presentation and Management. Circulation 1997; 95: 2098-107.

28. Olaison L, Petterson G. Current best practices and guidelines: Indications for surgical intervention in infective endocarditis. Infect Dis Clin North Am 2002; 16: 453-75.

29. Vikram HR, Buenconsejo J, Hasbún R, Buenconsejo J, Quagliarello VJ. Impact of valve surgery on 6-month mortality in adults with complications; Left sided native valve endocarditis: A propensity analysis. JAMA 2003; 290: 3207-14.

30. Thuny F, Beurtherel S, Mancini J, Gariboldi V, Casalta JP, Riberi A, et al. The timing of surgery influences mortality and morbidity in adults with severe complicated infective endocarditis: a propensity analysis. Eur Heart J 2011; 32 (16): 2027-33.

31. Oyonarte M, Montagna R, Braun S, Maiers E, Rojos P, Cumsille JF. Endocarditis Infecciosa: Morbimortalidad en Chile: Resultados del Estudio Cooperativo Nacional de Endocarditis Infecciosa (ECNEI: 1998-2002). Rev Med Chile 2003; 131: 237-50.

32. Oyonarte M, Montagna R, Braun S, Rojo P, Jara JL, Cereceda M, Morales M, et al. Experiencia en 506 pacientes del Estudio Cooperativo Nacional de Endocarditis Infecciosa (ECNEI) y sobrevida a 10 años, 1998-2008. Rev Chil Cardiol 2008; 27: (Abstract) 442.

33. Oyonarte M, Braun S, Maiers E, Frank R, Labarca J. Manejo de la Endocarditis Infecciosa. Recomendaciones del Comité de Educación Continua de la Sociedad Chilena de Cirugía y Cirugía Cardiovascular. Rev Chil Cardiol 1996; 15: 185-9.

34. Moreillon P, Que Y. Infective endocarditis. Lancet 2004; 363: 139-49.

35. Beynon RP, Bahl VK, Prendergast BD. Infective endocarditis Br Medical J 2006; 333: 334-9.

36. Oyonarte M, Rojo P, Estévez A, Solis I, Akel C, Klenner C, Cumsille JF. Endocarditis Infecciosa: Factores pronósticos de mortalidad en 131 casos. Rev Med Chile 1997; 125: 165-73.

37. Castillo JC, Anguita MP, Ramírez A, Sles JR, Mesa D, Franco M, et al. Long term outcome of infective endocarditis in non-drug addict patients: A 10 year study. Heart 2000; 83: 525-50.

38. Ferreiros E, Nacinovich F, Casabé JH, Modenesi JC, Swieszkowski S, Cortés C, et al. Epidemiologic, clinical, 
and microbiologic profile of infective endocarditis in $\mathrm{Ar}$ gentina: A national survey. The infectious endocarditis en la República Argentina-2 (EIRA-2) Study. Am Heart J 2006; 151: 545-52.

39. Heiro M, Helenins H, Mâkilâ S, Hohenthal U, Savunen T, Engblom E, et al. Infective endocarditis in a Finnish Teaching Hospital: A study on 326 episodes treated during 1980-2004. Heart 2006; 92: 1457-62.

40. Hoen B, Alla F, Selton-Suty C, Béguinot I, Bouvet A, Briançon S, et al. Associaton pour l'Etude et la prevention de l' Endocardite infectieuse (AEPEI) study group. Changing the profile of infective endocarditis: results of a 1-year survey in France. JAMA 2002; 288: (1) 75-81.

41. Sanabria J, Alpert JS, Goldberg R, Pape LA, Cheeseman $\mathrm{SH}$. Increasing frequency of staphylococcal infective endocarditis: Experience at a university hospital, 1981 through 1988. Arch Intern Med 1990; 150 (6): 1305-9.

42. Benito N, Miró JM, de Lazzari E, Cabell CH, del Río A, Altclas J, et al. Health Care Associate Native Valve Endocarditis: Importance of Non-nosocomial Acquisition. Ann Intern Med 2009; 150: 586-94.

43. Braun S, Escalona A, Chamorro G, Corbalán R, Pérez C, Labarca J, et al. Endocarditis Infecciosa: Análisis de 261 casos y resultados del tratamiento con un enfoque multidisciplinario. Rev Med Chile 2000; 128: 708-20.

44. Nunes MC, Gelape C, Ferrari T. Profile of infective endocarditis at a tertiary center in Brazil during a seven-year period: prognostic factors and in-hospital outcome. Int Journal of Infectious Dis 2010; 14: e394-8.

45. Hoen B, Selton-Suty C, Lacassin F, Etienne J, Briançon $\mathrm{S}$, Leport $\mathrm{C}$, et al. Infective endocarditis in patients with negative blood cultures: Analysis of 88 cases from a 1-year nationwide survey in France. Clin Infect Dis 1995; 20: 501-6.

46. Lafourcade M, García P, Thompson L. Microbiología en Endocarditis Infecciosa. Análisis crítico de la bacteriología en Chile. El desafío de los hemocultivos negativos. Rev Chil Cardiol 2004; 23 (2): 1118.

47. Rice A P, Madico EG. Polymerase Chain Reaction to diagnose Infective Endocarditis: Will it replace blood cultures? Circulation 2005; 111: 1352-4.

48. Shin GY, Manuel RJ, Gori S, Brecker S, Breathnach AS. Molecular technique identifies the pathogen responsible for a negative culture in infective endocarditis. Heart 2005; 91: 47-54.

49. Dalahaye F, Célard M, Roth O, de Gevigney G. Indications and optimal timing of surgery in infective endo- carditis. Heart 2004; 90: 618-20.

50. Farzaneh-Far R, Bolger AF. Surgical timing in Infective Endocarditis: Wrestling with the Unrandomized. Circulation 2010; 121: 960-2.

51. Lalani T, Cabell HC, Benjamín DK, Lasca O, Naber C, Fowler UG, et al. Analysis of the impact of early surgery in the cases of in-hospital mortality of Native Valve Endocarditis. Use of propensity score and Instrumental variable methods to adjust for treatment selection Bias. Circulation 2010; 121: 1005-13.

52. Prendergast B, Tornos P. Surgery for Infective Endocarditis. Who and When? Circulation 2010; 121: 1145-52.

53. Botelho-Nevers E, Thuny F, Casalta JP, Richet H, Gouriet F, Collart F, et al. Dramatic reduction in Infective Endocarditis-related mortality with a management-based approach. Arch Intern Med 2009: 169 (14): 1290-8.

54. Kemose HH, Lund O, Zollinger, Krommann A, Hansen O. Risk factors for early and late outcome after surgical treatment of native infective endocarditis. Scan J Thorac Cardiovasc Surg 1990; 24: 11-20.

55. Tornos MP, Permanyer-Miralda G, Olona M, Gil M, Galve E, Almirante B, Soler-Soler J. Long term complications of native valve Infective endocarditis in non addicts. A 15-year follow-up study. Ann Intern Med 1992; 117: 567-72.

56. Netzer ROM, Altwegg SC, Zollinger E, Tâuber M, Carrel T, Seiler C. Infective endocarditis: Determinants of long term outcome. Heart 2002; 88: 61-6.

57. Hasbún R, Holenarasipur RV, Barakat L, Buenconsejo J, Quagliarello V, et al. Complicated Left Sided Native Valve Endocarditis in Adults. Risk classification for mortality. JAMA 2003; 289: 1933-40.

58. Tornos P, González- Alujas T, Thuny F, Habib G. Infective Endocarditis: The European Viewpoint. Curr Probl Cardiol 2011; 36: 175-222.

59. Zalaquett R, Garrido L, Casas F, Morán S, Irarrázaval M, Becker $\mathrm{P}$, et al. Cirugía valvular reparadora en Endocarditis Infecciosa. Rev Med Chile 2004; 132: 307-15.

60. Bahamondes JC, Meriño G, Silva A, Salman J, Redel I, Droguett JP. Cirugía de la Endocarditis Infecciosa en un centro Regional en Chile. Análisis de casos y resultados en el largo plazo. Rev Med Chile 2008; 136: 31-7.

61. Seguel E, González R, Alarcón E, Stockins A, Neira L, Aguilera D, Carreño F. Factores asociados a mortalidad en cirugía de la Endocarditis Infecciosa en el Hospital regional de Concepción entre 1985 y 2005. Rev Chil Cardiol 2008; 27 (4): 470-9. 